\title{
Vestibular Information Is Required for Dead Reckoning in the Rat
}

\author{
Douglas G. Wallace, Dustin J. Hines, Sergio M. Pellis, and lan Q. Whishaw \\ Canadian Centre for Behavioural Neuroscience, University of Lethbridge, Lethbridge, Alberta, Canada T1K 3M4
}

Dead reckoning is an on-line form of spatial navigation used by an animal to identify its present location and return directly to a starting location, even after circuitous outward trips. At present, it is not known which of several self-movement cues (efferent copy from movement commands, proprioceptive information, sensory flow, or vestibular information) are used to compute homeward trajectories. To determine whether vestibular information is important for dead reckoning, the impact of chemical labyrinthectomy was evaluated in a test that demanded on-line computation of a homeward trajectory. Rats were habituated to leave a refuge that was visible from all locations on a circular table to forage for large food pellets, which they carried back to the refuge to eat. Two different probe trials were given: (1) the rats foraged from the same spatial location from a hidden refuge in the light and so were able to use visual cues to navigate; (2) the same procedure took place in the dark, constraining the animals to dead reckon. Although control rats carried food directly and rapidly back to the refuge on both probes, the rats with vestibular lesions were able to do so on the hidden refuge but not on the dark probe. The scores of vestibular reflex tests predicted the dead reckoning deficit. The vestibular animals were also impaired in learning a new piloting task. This is the first unambiguous demonstration that vestibular information is used in dead reckoning and also contributes to piloting.

Key words: dead reckoning; spatial navigation and dead reckoning; spatial navigation and vestibular system; vestibular system and dead reckoning; vestibular reflexes; food hoarding
Animals may navigate by piloting and dead reckoning (Gallistel, 1990). Piloting relies on external (allothetic) cues: visual, auditory, or olfactory. It is thought to be used by laboratory animals when performing most T-mazes (Rawlins and Olton, 1982), radial arm mazes (Olton et al., 1979; Jarrard, 1983), or water-based swimming pool spatial tasks (Morris et al., 1982; Sutherland et al., 1982). Dead reckoning is a form of on-line navigation that relies on self-movement (idiothetic) cues. First suggested by Darwin (1873) to be a form of navigation, dead reckoning has been demonstrated in many laboratory tests by removing allothetic cues from a testing situation (Mittelstaedt and Mittelstaedt, 1980; Etienne et al., 1986; Seguinot et al., 1993; Maaswinkel et al., 1999; Whishaw and Gorny, 1999). It is presently unknown which of several self-movement cues (vestibular information, proprioceptive information, sensory flow, or efferent copy from movement commands) are used for dead reckoning.

There are three reasons to believe that the vestibular system may be important for dead reckoning. First, signals concerning linear and angular acceleration could be integrated to indicate a present position in relation to a starting point (Barlow, 1964; Potegal, 1982). Double integration of the signals would allow a direct return to a starting point. Second, electrophysiological studies implicate the vestibular system as a source of information for spatial guidance (Wiener and Berthoz, 1993; Sharpe et al., 1995; Stackman and Taube, 1997; Taube, 1998; Russell et al., 2000). Third, a number of studies have suggested that vestibular information contributes to learned directional calculations (Potegal et al., 1977; Miller et al., 1983; Matthews et al., 1989; Semenov

\footnotetext{
Received May 22, 2002; revised Aug. 30, 2002; accepted Sept. 8, 2002.

This research was supported by grants from the Canadian Institute of Health Research.

Correspondence should be addressed to Douglas G. Wallace, Canadian Centre for Behavioural Neuroscience, University of Lethbridge, Lethbridge, Alberta, Canada T1K 3M4. E-mail: douglas.wallace@uleth.ca.

Copyright (C) 2002 Society for Neuroscience 0270-6474/02/2210009-09\$15.00/0
}

and Bures, 1989; Chapuis et al., 1992; Stackman and Herbert, 2002).

To determine which self-movement cues contribute to dead reckoning, it is essential to use a task in which an on-line calculation is computed using just completed movements (Mittelstaedt and Mittelstaedt, 1980). Thus, the response is unlearned and trial unique, and the dead reckoning calculation is made on-line from a circuitous outward trip; this is in contrast to directional calculations in which animals require training over multiple sessions. The purpose of the present experiment was to design such a task and to unambiguously assess the role of vestibular information in dead reckoning by showing that animals with vestibular lesions are unable to perform such an idiothetic navigation task but still able to perform a similar allothetic navigation task.

The experimental design had control rats and rats with sodium arsanilate-induced vestibular lesions forage from a home base for a large food pellet, located somewhere on the surface of a circular table, that they carried back to the refuge to eat (Whishaw and Tomie, 1997). The refuge was cued by a black box positioned over the home base and was clearly visible to a rat from the table surface. It was predicted that if the cued refuge were replaced by a hidden refuge in the light, then both groups of rats would be able to pilot using ambient visual cues to return to the starting location (Whishaw and Mittleman, 1986). If the probe trial were given in complete darkness, however, the control rats would dead reckon using idiothetic cues generated on the just completed outward search, but the vestibular group would be impaired. The same animals were used to demonstrate that an impairment displayed by vestibular rats in a new allothetic spatial problem implies that vestibular information contributed to dead reckoning cues as well as to learning new piloting problems.

\section{MATERIALS AND METHODS}

Subjects. The subjects were 23 female Long-Evans rats (University of Lethbridge vivarium) weighing $\sim 250-300 \mathrm{gm}$. Rats were housed in 
groups in wire mesh cages. The colony room was maintained at $20-21^{\circ} \mathrm{C}$ with a $12 \mathrm{hr}$ light/dark cycle. Ten rats received bilateral labyrinthectomies 2 weeks before the start of testing, and seven rats served as controls. The remaining six rats received unilateral vestibular lesions.

Surgeries. Animals were deeply anesthetized with sodium pentobarbital (Somnotol; MTC Pharmaceuticals, Cambridge, Ontario, Canada). Labyrinthectomies were produced by giving rats intratympanic injections of sodium arsanilate (Sigma, Oakville, Ontario, Canada). Ten rats received bilateral labyrinthectomies, in which each ear received $20 \mathrm{mg} / \mathrm{kg}$ sodium arsanilate $(100 \mathrm{mg} / \mathrm{ml}$ sodium arsanilate in $0.9 \%$ saline $)$ as described by Chen et al. (1986). Subsequent to injection of sodium arsanilate, each ear canal was packed with Gelfoam (Upjohn, Kalamazoo, MI). Six rats received unilateral labyrinthectomies in which only one ear received 20 $\mathrm{mg} / \mathrm{kg}$ sodium arsanilate $(100 \mathrm{mg} / \mathrm{ml}$ sodium arsanilate in $0.9 \%$ saline $)$ and was subsequently packed with Gelfoam. The unilateral labyrinthectomized rats were included as a baseline to evaluate the extent to which our bilateral labyrinthectomies actually produced bilateral damage to the vestibular system [the magnitude of damage associated with a unilateral labyrinthectomy can been seen in the histology of Chen et al. (1986)]. The rats' auditory ability was not assessed.

Feeding. After rats had recovered from the labyrinthectomy, they were maintained at $85 \%$ of their ad libitum feeding weight during the course of the experiment. Throughout the experiment, rats searched for randomly located, large $(750 \mathrm{mg})$ food pellets (Bio-Serv, Frenchtown, NJ). Rats have been found to reliably carry these food pellets to the refuge (Whishaw et al., 1995). After testing each day, rats were fed LabDiet Laboratory Rodent Pellets in their home cage.

Apparatus. The apparatus (Fig. 1) was a large circular table $(205 \mathrm{~cm}$ in diameter) similar to that described by Barnes (1979) [see also Whishaw and Tomie (1997)]. The table was mounted on ball bearings; therefore, it could be rotated between animals permitting the displacement of any odor cues. Eight holes $(11.5 \mathrm{~cm}$ in diameter) were arranged at an equal distance around the perimeter of the table. The table was located $75 \mathrm{~cm}$ above the floor in a large room with many cues, including a refrigerator, desk, computer, cupboards, and chairs (Fig. 1, top). The testing room was lightproof such that, when all of the lights were turned off, no light was present in the room. The experimenter wore infrared goggles to observe the animal during dark testing.

The home base was a Plexiglas box $(20 \times 29 \times 22 \mathrm{~cm})$ that had runners so that it could be affixed below any of the holes on the table. The cue box was identical to the home base except that there was a hole $(11.5 \mathrm{~cm}$ in diameter) in the shorter side, thereby permitting the animals to climb up onto and explore the table and then exit through the hole located on the side of the box.

Habituation. Rats were habituated to the table and trained to find randomly located food pellets. Habituation involved allowing rats to find and carry food pellets to a cued home base. Subsequent to habituation, vestibular lesions were given, and rats received additional hoarding experience. Rats were habituated with the cued home base and received occasional trials with an uncued home base and under both light and dark conditions. The additional habituation under place and dark conditions reduced the surprise associated with the changed testing conditions associated with probe trials.

Testing procedures. Rats were given a series of baseline training and probe sessions. In general, rats received several days of baseline training followed by a day with one probe trial. This continued until all rats were exposed to each probe trial twice. The following sections describe the procedures used during each phase of testing.

Baseline cued testing. Rats left a cued refuge and searched the table for a randomly located food pellet. The cue was a refuge cage placed above the hole under which the refuge was located. A rat was always released from the same location with respect to the room, but for each rat, that location was different. During each daily session, rats were given four trials (one possible set of food pellet locations is shown in Fig. 1A). One trial consisted of the rat leaving the cued home base, finding the food, and then returning to the home base (i.e., cued task) (Fig. 1A). After completion of the first trial, while the rat was still in the refuge, a second food pellet was placed on the table in a random location, etc. Rats foraged for $5 \mathrm{~d}$ before receiving a probe trial.

Place probe-light. Both groups were required to search for one food pellet on the table, with the cage that marked the location of the home base absent (i.e., place probe) (Fig. $1 B$ ). After the rats found the food pellet and returned to the home base, testing was concluded for that day. Elimination of the proximal cue restricted the rats to using either distal allothetic cues or self-movement cues.
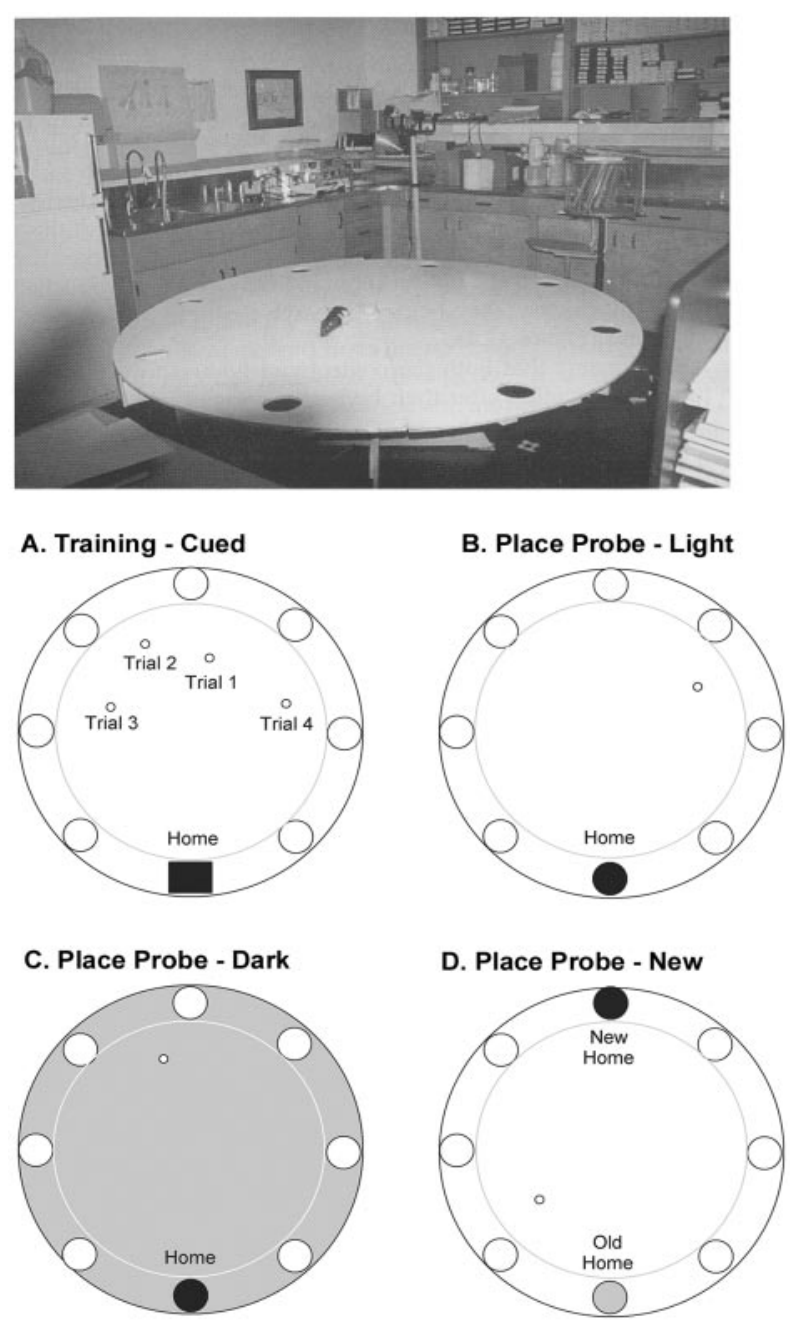

Figure 1. Test room and test procedures. Top, The test room. Many visual cues are available to the rat under light conditions; in the dark, visual cues are eliminated. $A$, Schematic of the table and a possible sequence of food pellet placements for $1 \mathrm{~d}$. Black square indicates cued home base. Rats were required to find each food pellet before the table was rebaited. $B, C$, Schematics of the place probe-light and place probedark. Black circle indicates hidden home base. $D$, Schematic of the place probe-new location. Gray circle indicates former location of the home base; black circle indicates new location of the home base. For all task components, the heading direction circle that is drawn tangent to the inner portion of the holes was used to code when a return trip was terminated, and the point at which the rat crossed the circle was used to calculate the rat's heading direction.

Place probe-dark. Both groups were required to search for one food pellet on the table from a hidden refuge with the lights turned off (i.e., dark probe) (Fig. 1C). After the rats found the food pellet and returned to the home base, testing was concluded for that day. This probe eliminated all visual cues, thereby restricting the rat to using idiothetic cues.

Place probe-new location. During the $2 \mathrm{~d}$ that followed the last place probe, rats were released from an uncued home base that was $180^{\circ}$ different from what was encountered during training and probes (i.e., new home location) (Fig. 1D). Each group was given four trials per day for $2 \mathrm{~d}$. This probe resulted in a conflict between allothetic information, learned during cued training (and the place probe-light), and idiothetic information, generated on the outward-bound trip, as cues for the location of the home base.

Analysis of spatial navigation. An HI-8 Sony video camera with infrared taping abilities was used to record the rat's movements during testing. Foraging trips generated by each rat were copied onto a transparency via an HI- 8 video player and TV monitor. The homeward segment of the trip 
was defined as the path generated by the rat after finding the food pellet until it crossed a circle that would be tangent to the inner portion of the holes at the periphery of the table (Fig. 1). Homeward trip segments were analyzed with circular statistics to determine the basis of group differences in heading direction error (Batschelet, 1981). Heading direction was calculated by measuring the angle between the line that connected the point where the food was found and the home base and the line between the point where the food was found and the point where the rat's path crossed the circle, described above. The home base was set at zero, with angles increasing in steps of $5^{\circ}$ counterclockwise around the perimeter of the table. A group's heading direction is a function of two parameters: (1) mean angle and (2) angular variance. The mean angle reflects the central tendency of a group's heading directions. Mean angles can range from 0 to $360^{\circ}$. Angular variance reflects the spread of a group's heading directions and is measured by the parameter of concentration. Parameter of concentration ranges from 0 (in which heading directions are randomly scattered around the perimeter of the table) to 1 (all heading directions are in the same direction). Mean angle and angular variance are evaluated for group differences under both testing conditions with the Watson-Williams test (Batschelet, 1981) and the parametric test for the concentration parameter (Batschelet, 1981), respectively.

Foraging trips to be analyzed for their kinematic components were converted from analog recording to a digital computer file of a Peak Performance (Peak Performance Ltd., Englewood, CO) system with a sampling rate of $60 \mathrm{~Hz}$. The foraging trip traveled by the rat is acquired from the digitized file by sampling the $x$ - and $y$-coordinates of the rat as it moves along its path. Acquiring the path involves manually tracking a single point on the animal (the middle of the back at the level of shoulders was selected as the point to track on each animal) by selecting one pixel per frame every 10 frames of the digitized video. The $x$ - and $y$-coordinate velocity is computed from the sampled raw distance data. The resultant velocity (meters per second) is computed from these data. The data reflect moment-to-moment velocities during one foraging trip. In previous work, we have used the point-of-inflection from the cumulative velocity distribution as a measure of central tendency for the rats' moment-to-moment velocities (Wallace et al., 2002). Briefly, a frequency distribution was constructed for the moment-to-moment velocities observed on the homeward trip segment. The frequencies were calculated by counting the number of velocities that fell within a specific range or bin. The frequency for each bin was then divided by the total number of moment-to-moment velocities yielding the proportion of each trip segment that was spent traveling at velocities that fell within that bin. Bins were $0.060 \mathrm{~m} / \mathrm{sec}$ in size, with the first bin ranging from 0.001 to 0.060 $\mathrm{m} / \mathrm{sec}$, the second bin ranging from 0.061 to $0.120 \mathrm{~m} / \mathrm{sec}$, the third bin ranging from 0.121 to $0.180 \mathrm{~m} / \mathrm{sec}$, and so on, until the maximum velocity of $0.96 \mathrm{~m} / \mathrm{sec}$. The frequency distribution of the homeward trip segment was transformed into a cumulative velocity distribution. Finally, pointsof-inflection were estimated for each rat's homeward trip segment by fitting a three-parameter sigmoidal function to the corresponding cumulative velocity distribution. Drai et al. (2000) used similar statistical procedures to characterize the speeds, or "gears," at which rats move through an environment.

Assessment of static and statokinetic reflexes. Vestibular function was assessed by measuring static and statokinetic vestibular reflexes [for a more complete discussion of static and statokinetic reflexes, see Monnier (1970)]. Five behavioral tests were used (Sirkin et al., 1980; Pellis et al., 1991, 1992; Pellis and Pellis, 1994). The static reflexes were tested to examine the capacity of the vestibular system to provide the information necessary to orient the head horizontally when stationary. Two types of head-righting reflexes were assessed. The first involved holding the rat laterally in the air. In this position, only the otoliths on the side facing the ground provide information on head position; that is, the reflex is asymmetrical. Hence, each side of the body can be tested separately. If the otoliths are intact, the head rotates around the longitudinal axis of the body so that the ventral surface of the head faces the ground. In the absence of otolith function, the head is not righted or is oriented skyward. The second test involved holding the rat upside down in the air by the base of the tail. In this case, because information from the otoliths on both sides is used, it is a symmetrical reflex. After release of the forepaws from contact with the ground, if the otoliths are functioning normally, the head is dorsiflexed: the head is oriented so that the ventral surface is horizontal to the ground. If the otoliths are damaged, the head is ventroflexed when the forepaws lose contact with the ground. When the otoliths are intact, the head may be ventroflexed, but only after it is
Table 1. Vestibular assessment scale

\begin{tabular}{llll} 
Reflex type & Behavioral test & Yes & No \\
\hline Static reflexes & Head righting & & \\
& Asymmetrical & & \\
& $\quad$ Right & 0 & 1 \\
& Left & 0 & 1 \\
& Symmetrical & & \\
& $\quad$ Dorsal flexion & 0 & 1 \\
Statokinetic reflexes & Linear acceleration & & \\
& Head & 0 & 1 \\
& Limbs & 0 & 1 \\
& Angular acceleration & & \\
& Right & 0 & 1 \\
& Left & 0 & 1 \\
& Air righting & & \\
& Complete & 0 & \\
& Partial & 1 & \\
None & 2 & \\
& Minimum & 0 & \\
& Maximum & 9 & \\
\hline
\end{tabular}

0, No vestibular damage; 9 , complete vestibular damage.

first dorsiflexed. Therefore, in both tests, the head is oriented horizontally to the ground if the vestibular apparatus is intact.

Three statokinetic reflex tests were used to assess the ability of the vestibular system to provide the information necessary to maintain a stable horizontal head orientation when the rat is in motion. Two tests were used to assess response to linear acceleration. In the first, the rats were held by the base of the tail and lowered toward the ground. Animals with intact semicircular canals flex their necks and extend their forelimbs as they approach the surface, whereas the rats with vestibular damage do so only when their forepaws or vibrissae touch the ground. In the second test, the animals were tested to assess their ability to right themselves in the air. During the air-righting test, the rats were held $\sim 50 \mathrm{~cm}$ above a cushion in an upside down position; the experimenter removed his/her hands as quickly and simultaneously as possible. With intact labyrinths, rats rotate cephalocaudally around their longitudinal axis; however, after vestibular damage, the righting response is abolished, or at least greatly curtailed. The final test was used to assess the response to angular acceleration. The rats were held by the mid body while they had all four paws in contact with the ground and rapidly rotated $90^{\circ}$ from the starting position, first in one direction and then the other. Rats with intact labyrinths moved their heads and necks in compensation to maintain their heads in the original orientation. This test was useful for assessing whether the semicircular canal damage was present to the same degree on both sides. The numerical scores given to each of the behavioral tests were summed to provide an index of vestibular damage. Scores of zero indicated no vestibular damage, and scores ranging from 1 to 9 showed increasing degrees of vestibular damage (Table 1). Static and statokinetic asymmetry indexes were calculated for both unilateral and bilateral animals. The static asymmetry index was calculated by finding the difference between the head-righting score observed for each side of the body. The statokinetic asymmetry index was calculated by finding the difference between the angular acceleration score observed for each side of the body.

\section{RESULTS}

\section{Habituation performance}

Initially, vestibular rats were hesitant to leave the home base relative to control animals. After continued habituation to the table, both control and vestibular animals found the food pellets and returned to the cued home base under normal light conditions. During habituation to place and dark procedures, vestibular animals were initially reluctant to leave the home base; however, after several sessions, the animals left the home base under each condition. 


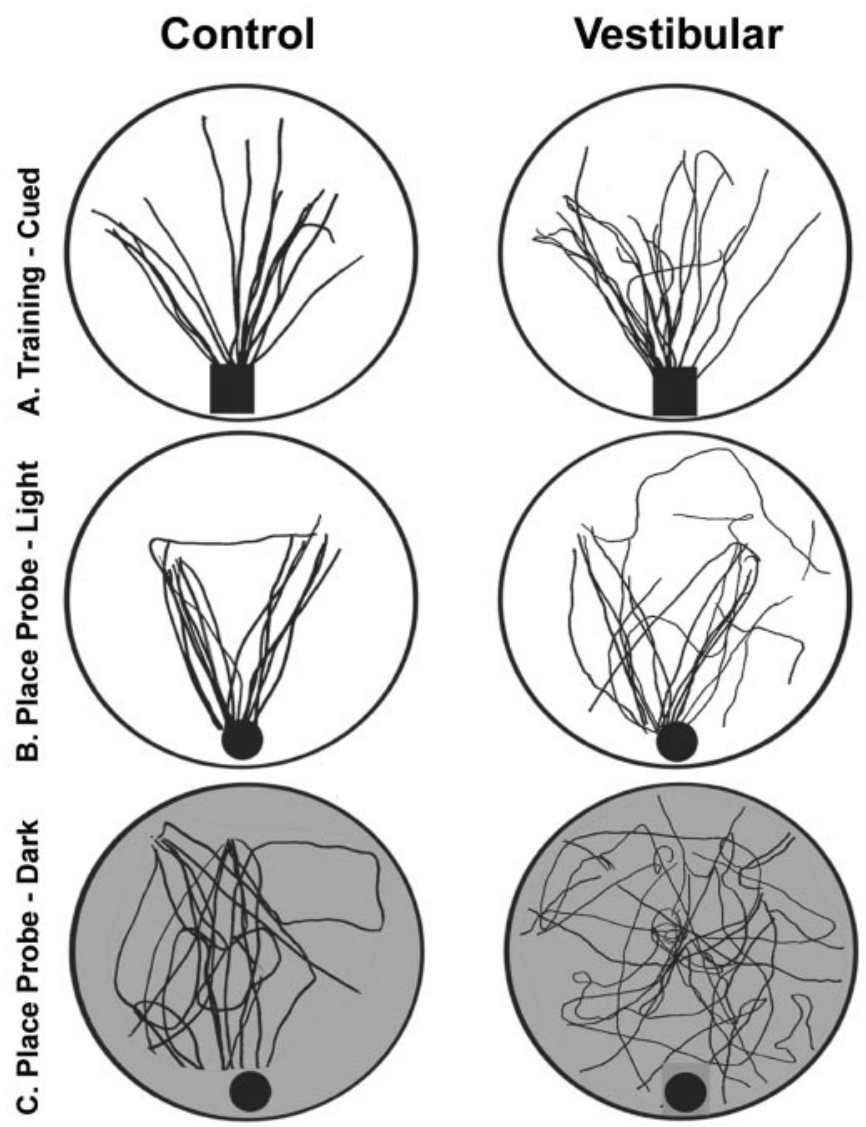

Figure 2. Homeward paths of control and vestibular rats. Each diagram plots the normalized homeward paths for a group of animals under one of the testing conditions: $A$, homeward paths with the home base cued (black square); $B$, homeward paths with the home base hidden (black circle); $C$, homeward paths in the dark. Note: Accuracy with respect to heading direction at the point where rats crossed the heading direction circle is shown in Figure 3.

\section{Homeward paths}

Figure $2 A$ plots each homeward trip for control (left) and vestibular (right) groups when the home base was cued (data normalized to a common start point). Both groups made direct paths back to the home base after finding the food pellet. Figure 2, $B$ and $C$, presents the homeward paths observed for both groups of rats tested on the place probe-light and place probe-dark, respectively. In general, control and vestibular rats' homeward paths were direct when the cue for the home base was removed; however, there were two paths from the vestibular rats that were outliers $(B)$. When all of the allothetic cues were removed, control animals still returned to the home base along a direct path, except for one outlier. In contrast, vestibular rats' homeward paths were less direct and more circuitous relative to controls.

\section{Heading direction}

The outer circle of Figure 3 plots the heading directions for two homeward paths under cued training, place probe-light, and place probe-dark from control and vestibular groups. The points on the inner circle represent the average heading direction from both homeward paths made by each rat under the training and probe conditions. Circular statistics were used to evaluate the basis of group differences in heading direction for the homeward segment of trips under each condition (Batschelet, 1981). The groups did not differ significantly in their mean angular heading direction during cued training, place probe-light, or place probedark (Watson-Williams test). Although groups did not differ significantly in their angular variance during cued training or the place probe-light, the vestibular group's average heading direction was significantly more variable than the control group's average heading direction on the place probe-dark $\left(F_{(9,6)}=\right.$ 20.33; $p<0.05$; parametric test for the concentration parameter). This pattern of results was consistent with the vestibular animals' nonsystematic error in heading direction, which was restricted to the place probe-dark.

\section{Kinematic analysis}

Figure 4 presents moment-to-moment velocity profiles and the corresponding spatial arrangement of a single foraging trip from a representative control and vestibular rat on cued training $(A)$, place probe-light $(B)$, and place probe-dark $(C)$. An ANOVA conducted on the point-of-inflection from the homeward portion of the trip under the cued training, place probe-light, and place probe-dark revealed a significant effect of probe $\left(F_{(2,30)}=\right.$ $16.072 ; p<0.05)$. The absence of significant group effect or group by probe interaction is consistent with preserved modulation of speed after the food pellet is found.

Figure 5, top panels, presents control and vestibular average times for the outward (left) and homeward (right) trip segments under each probe. The ANOVA conducted on the observed times revealed a significant effect of probe $\left(F_{(2,30)}=22.822 ; p<0.05\right)$, with both trip segment and group factors not significant. The group by trip segment interaction $\left(F_{(1,15)}=10.328 ; p<0.05\right)$ and the group by trip segment by probe interaction $\left(F_{(2,30)}=6.719\right.$; $p<0.05)$ were significant, whereas other interactions were not found to be significant. Post hoc analysis revealed that vestibular animals took significantly longer to return to the home base than control animals only under the dark probe (Tukey LSD; $p<$ $0.05)$.

A similar analysis was conducted on trip segment distances (Fig. 5, bottom panels). The ANOVA conducted on trip segment distance revealed a significant effect of probe $\left(F_{(2,30)}=11.035\right.$; $p<0.05)$, with both trip segment and group factors not significant. Trip segment by group $\left(F_{(1,15)}=8.498 ; p<0.05\right)$, probe by group $\left(F_{(2,30)}=4.558 ; p<0.05\right)$, trip segment by probe $\left(F_{(2,30)}=\right.$ $6.053 ; p<0.05)$, and trip segment by probe by group $\left(F_{(2,30)}=\right.$ 4.115; $p<0.05)$ interactions were significant. Post hoc analysis revealed that the homeward path of the vestibular animals was significantly longer than that of the control animals only under the dark probe (Tukey LSD; $p<0.05$ ).

\section{New refuge location}

When the rats were released from a location $180^{\circ}$ different from training and probes, the number of returns to the old home base location and the sequence of hole selections were recorded. Figure 6 plots the average number of returns to the old refuge location across both days of testing. The ANOVA conducted on the average number of perseveration responses across both days of testing revealed a significant effect of day $\left(F_{(1,10)}=20.028 ; p<\right.$ $0.05)$ and a significant group by day interaction $\left(F_{(1,10)}=5.921\right.$; $p<0.05)$. The effect of group was not found to be significantly different. Post hoc analysis revealed that vestibular animals had significantly more returns to the old home base location during both blocks of training relative to controls. In addition, only the vestibular group demonstrated a significant decrease in perseveration across days (Tukey LSD; $p<0.05$ ). 


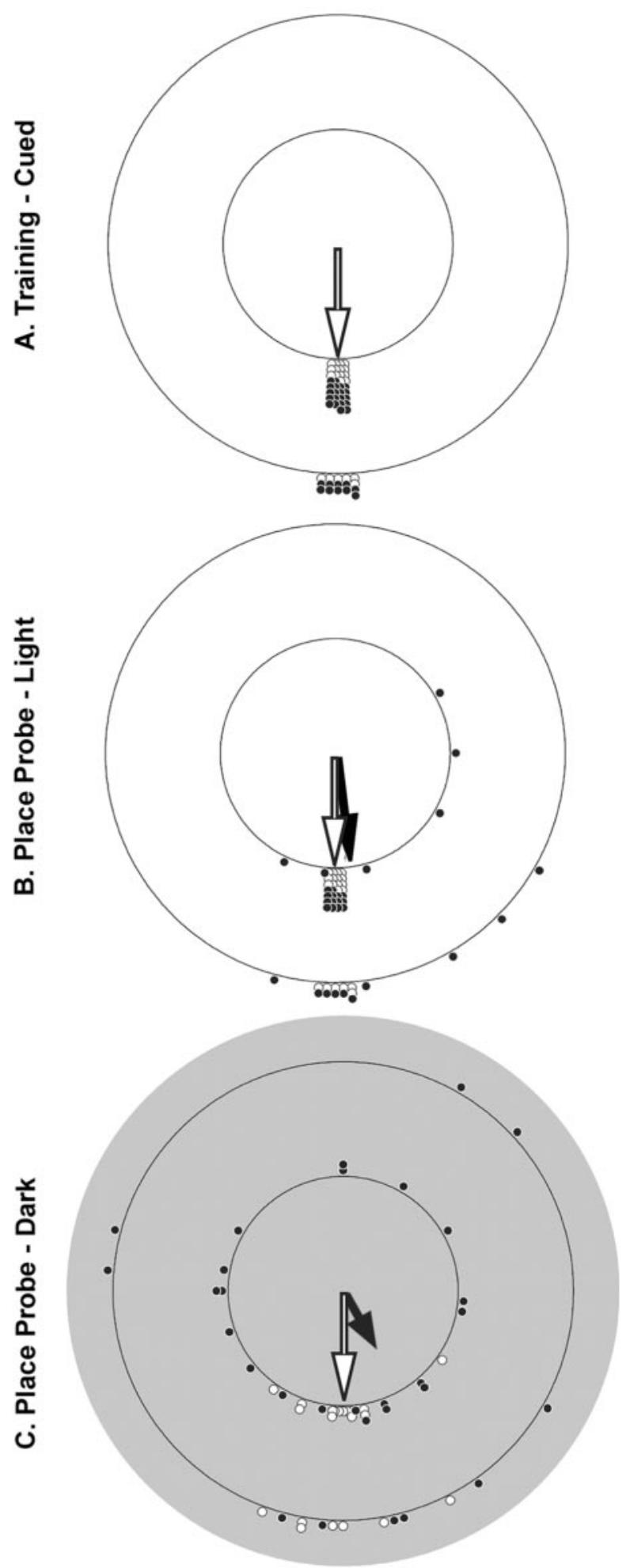

Figure 3. Circular statistics for control and vestibular animals illustrating the poor performance of vestibular animals in the dark test. The three testing conditions are as follows: $A$, training with the home base cue; $B$, place probe with the home base hidden; $C$, place probe in the dark. White dots represent control animals; black dots represent vestibular animals. Inner circle represents raw data; outer circle represents averaged data. White arrows point to control average heading direction and parameter of concentration. Black arrows point to vestibular average heading direction and parameter of concentration. The bottom of the circular plot corresponds to normalized location of the home base, with heading direction angles increasing from 0 to $360^{\circ}$ in a counterclockwise direction. The
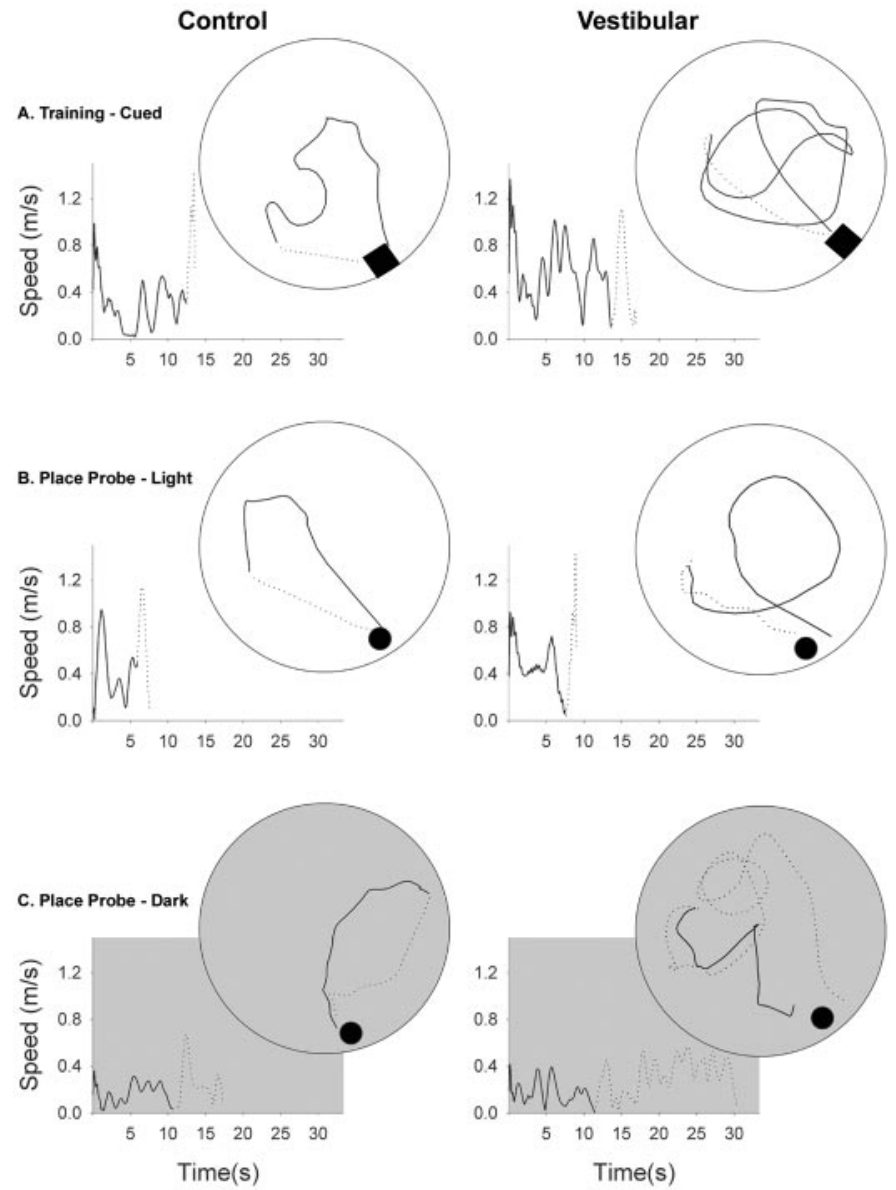

Figure 4. Representative control and vestibular animals' kinematic profiles. Outward paths are shown by solid lines; homeward paths are shown by the dotted line in both the representation of the rat's trip (circle) and its speed (graph). The three testing conditions are as follows: $A$, training with the home base cued; $B$, place probe with the home base hidden; $C$, place probe in the dark. Note: The vestibular animal's return trips are only longer and more nondirect in the dark condition.

When released from the new location, control and vestibular animals also differed in their sequence of hole visits. Figure 7 presents the first two hole visits from control (left) and vestibular (right) animals when released from a new location. The second choice was defined as the second hole that the rat selected other than the first hole selected by the rat. In general, both the control $(r=0.71 ; p<0.05$; Rayleigh test $)$ and vestibular $(r=0.75 ; p<$ 0.05 ; Rayleigh test) rats' first choices were directed toward the previous location of the home base. The control rats' second choice was directed primarily at the new location of the home base $(r=0.92 ; p<0.05$; Rayleigh test), with three rats selecting holes adjacent to the correct hole. Rats with vestibular lesions made second choices that were distributed randomly around the table ( $r=0.04 ; p>0.05$; Rayleigh test). In general, both groups returned to the old home base location first. Although the control

$\leftarrow$

length of the arrow corresponds to the group's parameter of concentration, whereas the direction of the arrow represents the group's average heading direction. An arrow that extends to the perimeter of the inner circle represents a parameter of concentration equal to 1 , with shorter arrows corresponding to lower values. Note: Only the vestibular animals are inaccurate under the dark condition. 


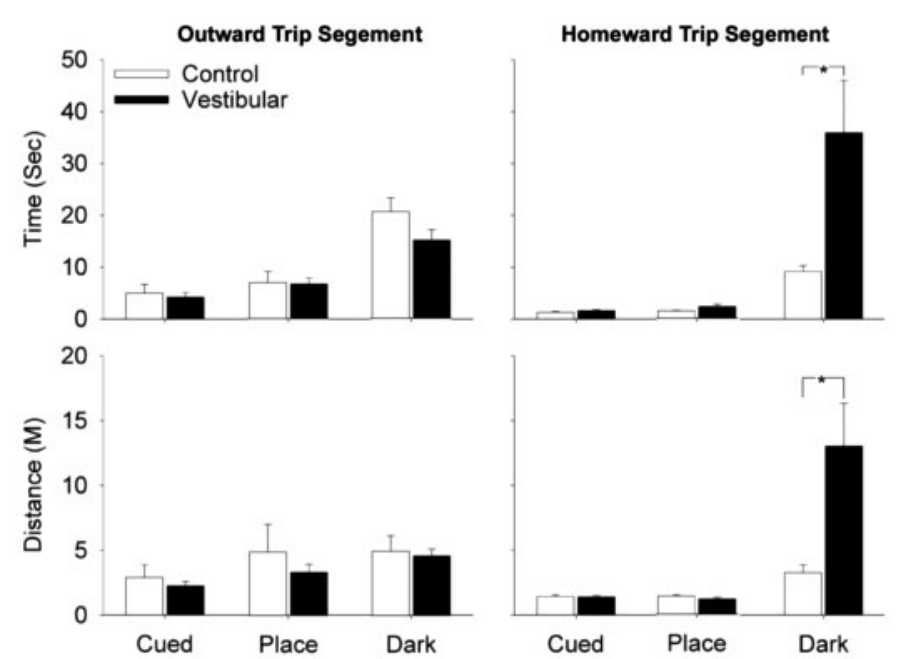

Figure 5. Exploratory trip distance and time (mean and SE). The left-hand panels plot control and vestibular average time (top) and distance (bottom) for the outward trip segment. The right-hand panels plot control and vestibular average time (top) and distance (bottom) for the homeward trip segment $\left({ }^{*} p<0.05\right.$; LSD test). Note: Elevated time and distance in the vestibular rats in the dark.

animals returned to the new home base second, animals with vestibular lesions were random on their second hole selection.

\section{Vestibular reflexes}

On the basis of the assessment of static and statokinetic reflexes, the rats varied in the degree of vestibular damage. Rats with unilateral vestibular lesions had significantly higher static $\left(t_{(14)}=\right.$ 4.836; $p<0.05)$ and statokinetic $\left(t_{(14)}=3.281 ; p<0.05\right)$ asymmetry indexes relative to rats with bilateral lesions. Subsequent analysis and discussion will be restricted to rats with bilateral lesions. To assess the relationship between vestibular damage and navigational abilities, we conducted several linear regressions. Figure 8 plots the data used in each analysis along with the regression line. The animal's score on the vestibular impairment scale was used to predict the parameter of concentration observed under cued training (Fig. 8A), place probe-light (Fig. 8B), and place probe-dark (Fig. $8 C$ ). In addition, we used the vestibular scores to predict the number of perseveration responses when the rats were released from a new location (Fig. 8D). Vestibular impairment was not a significant predictor for heading direction reliability (i.e., parameter of concentration) under cued training or the place probe-light. Both parameter of concentration during the place probe-dark and the number of perseverations were significantly predicted by performance on the vestibular scale. Rats with more severe vestibular damage had decreased heading direction reliability $\left(F_{(1,15)}=8.42 ; p<0.05\right)$ and an increased number of perseverations $\left(F_{(1,15)}=7.86 ; p<0.05\right)$. Vestibular damage was a significant predictor of behavior when the only source of information available was idiothetic cues. In contrast, when allothetic cues were available, performance was unrelated to vestibular damage.

\section{DISCUSSION}

This study examined the hypothesis that vestibular signals are required for dead reckoning. Control and bilaterally vestibularlesioned rats searched in the light for food pellets on a circular table and carried them directly back to a cued refuge for eating. When the cue to the refuge was removed, the homeward accuracy

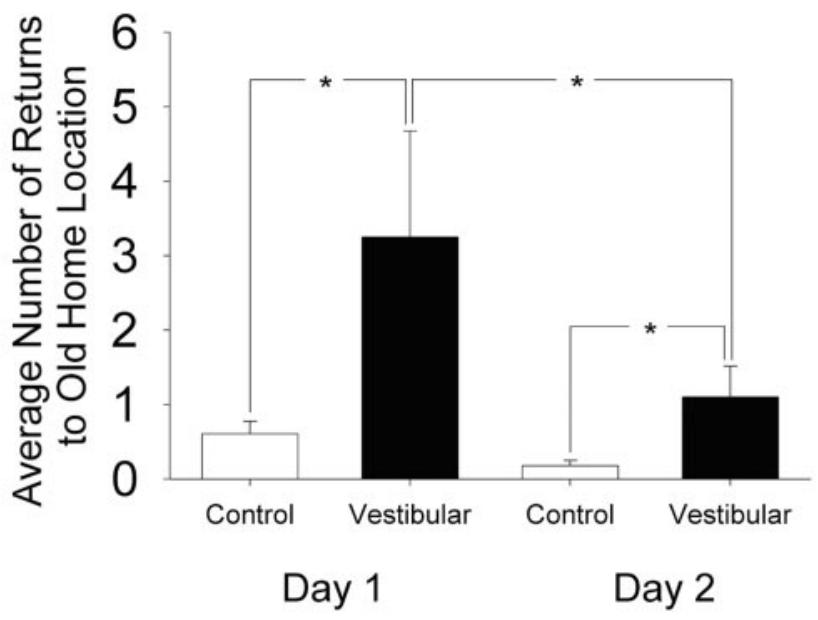

Figure 6. Number of returns (mean and SE) to the old home base location for control and vestibular groups on $2 \mathrm{~d}$ of testing with the home base in a new location $\left({ }^{*} p<0.05\right.$; LSD test).

of both groups was unchanged. When the lights were turned off, thereby preventing piloting by removing allothetic cues, control rats, but not vestibular rats, returned directly to the home base. This finding shows that navigating by dead reckoning depends on vestibular information. When the two groups of rats were presented with a new problem in the light, both groups demonstrated that they could use distal environmental cues to return to the former location of the home base. Subsequent to visiting the former home base location, control rats returned to the new home base location. In contrast, the vestibular-lesion rats' second hole choice was random, suggesting that dead reckoning also plays a role in spatial learning and contributes to new piloting-based spatial navigation.

The food-carrying behavior of foraging rats lends itself to the study of dead reckoning (Whishaw and Tomie, 1997). Foraging for randomly located food pellets requires a search that inevitably results in a circuitous outward trip that will vary from trial to trial. To return to the home base, an animal must either pilot with respect to allothetic cues or dead reckon using recently experienced idiothetic cues. In the present study, the strategy of having the rat return to a visible refuge ensured that the habituation component of the task could be performed by both control and vestibular-lesion rats. This form of beacon navigation is unlikely to require vestibular cues (O'Keefe and Nadel, 1978). In addition, both vestibular and control rats performing this task would also become familiar with the location of the home base in relation to ambient visual cues (Whishaw and Mittleman, 1986; Whishaw et al., 2001). The strategy of habituating the rats to both light and dark conditions from various starting locations ensured that the manipulations made on the probe trials would not be disruptive to the rats. In short, the methods that were used provided an unambiguous opportunity for the rats to dead reckon on the dark probe trial; thus they were a definitive test of the putative role of vestibular information in dead reckoning.

The finding that the rats with vestibular lesions were impaired when tested in the place probe-dark and not the place probelight, whereas the control rats were equally accurate in both conditions, demonstrates that vestibular information is essential for dead reckoning. It was not simply a difference in accuracy, however, that indicated that vestibular information contributes to dead reckoning. The behavior of the control rats was distinctive 

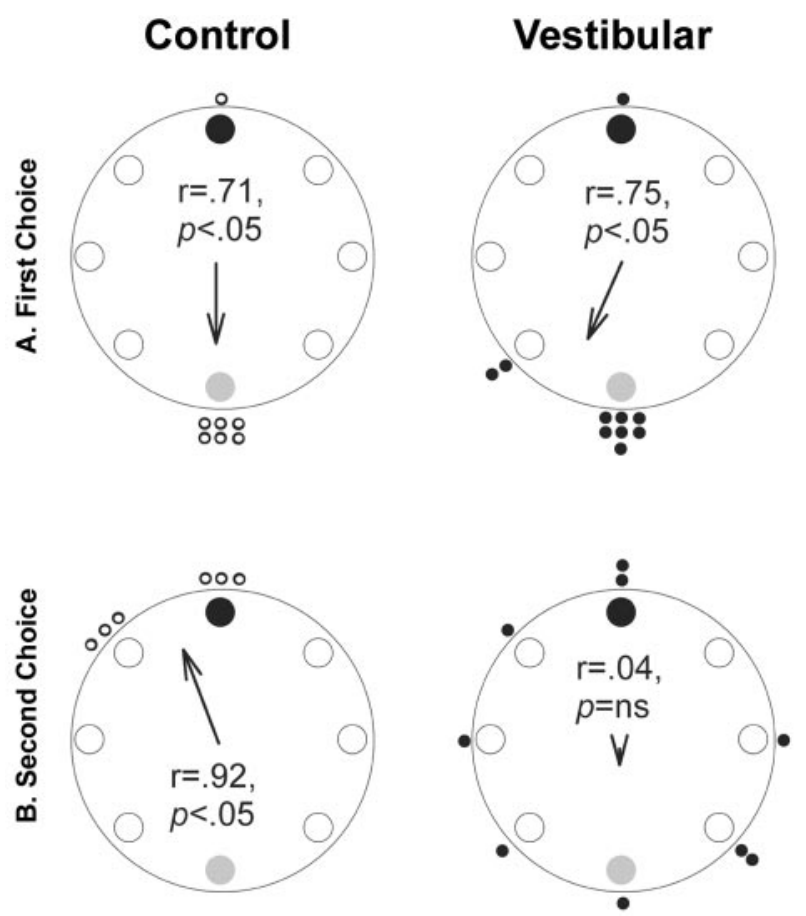

Correct Home

Previous Home Location

Figure 7. Group hole visits when released from a new home base location. $A$, Location of the first hole selected. $B$, Location of the second hole selected. Each plot is a diagram of the table with the correct location of the home base, previous location of the home base, and other home base locations indicated by black, gray, and white circles, respectively. Hole visits are represented by the black dots located around the outside of the circular diagram. The black arrows indicate average heading direction and parameter of concentration. The length and direction of the black arrow at the center of each graph corresponds to a group's parameter of concentration and average heading direction, respectively. The $r$ values and associated probabilities reflect the results of each plot from the Rayleigh test of randomness.

and similar in both light and dark probe conditions. After locating the food, they picked it up and then accelerated in a direct trajectory to the home base, without a stop. The homeward trip was thus different from the meandering outward trip that was characterized by pauses and head scans. The rats with vestibular lesions displayed meandering outward searches and direct homeward trips that, in their details, were very similar to those of the control rats in the light. On the dark probe, however, the vestibular rats picked up the food and accelerated, but their movement direction was random relative to the home. After an initial failed return, they stopped, made head scans or turns, proceeded in another direction, and continued in this way until they finally reached the home base, still carrying the food pellet. The fact that the vestibular rats persisted in their search for the home base and did not eat the food pellet on the table surface indicates that they were motivated to make the homeward trip. Thus, their behavior supports the interpretation that vestibular information is necessary for the dead reckoning calculation necessary for making a direct homeward trip in the dark.

Rats with unilateral lesions had significantly higher static and statokinetic asymmetry index scores relative to bilateral rats. These results provided evidence that the chemical labyrinthectomies were effective in disrupting both vestibular systems in rats receiving bilateral lesions.

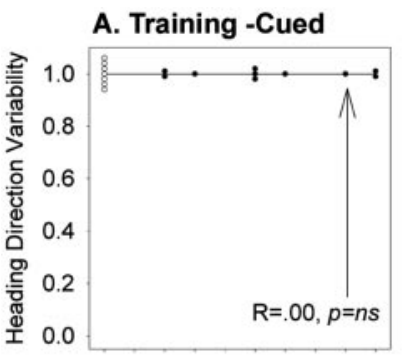

$0 \begin{array}{lllllllll}0 & 2 & 3 & 4 & 6 & 7 & 8 & 9\end{array}$

C. Place Probe - Dark
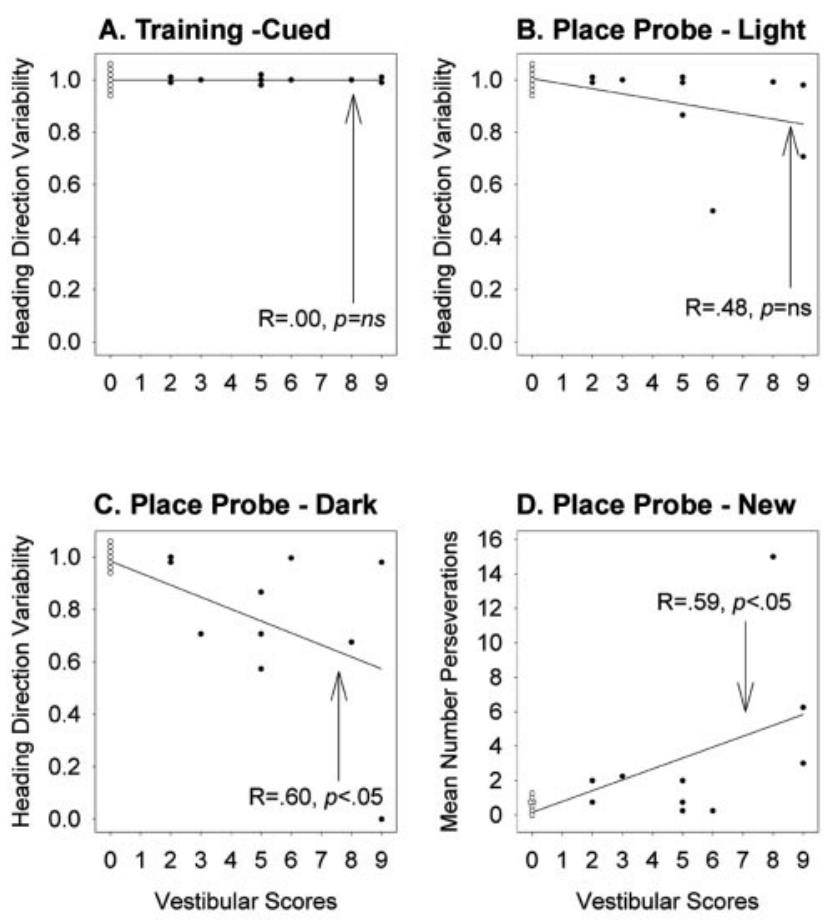

Figure 8. Regression analyses using vestibular reflex impairments as a predictor of spatial performance. White dots represent control animals; black dots represent vestibular animals. The four testing conditions are as follows: $A$, training with the home base cued; $B$, place probe with the home base hidden; $C$, place probe in the dark; $D$, place probe with the hidden home base in a new location. Spatial performance in $A-C$ was indexed by the parameter of concentration (i.e., variability of heading direction). Spatial performance in $D$ was indexed by mean number of perseverations to the old home base location.

An important confirmatory finding in the present study was the finding that the degree of vestibular impairment, as indicated by reflex tests, was significantly related to the dead reckoning deficit displayed by the rats. This finding demonstrates that as vestibular deficits become more severe, so too do the impairments in dead reckoning. The present results are consistent with earlier reports by Mittelstaedt and Mittelstaedt (1980) showing that subthreshold vestibular passive displacement in normal Mongolian gerbils returning home with a pup displaced their homeward journey. The strength of the present study, however, is the direct demonstration that accurate home returns unambiguously depend on vestibular information, as opposed to proprioceptive information. Furthermore, although the cries of the displaced pup no doubt resulted in linear outward trips, the foraging demands of the present study required circuitous outward trips, thus demonstrating that the accuracy of a homeward trip depended on calculations derived from angular and linear integrations of the circuitous outward path.

The results of a number of studies are consistent with the suggestion that the hippocampal formation (hippocampus and associated structures and pathways) may use vestibular cues to assist in spatial navigation. Control rats organize their exploration trips into circuitous outward and direct homeward components (Techernichovski et al., 1998; Drai et al., 2000). This work has led to the discovery that damage to the fimbria-fornix changes the organization of a rat's exploratory trips (Wallace et al., 2002). A rat's return to the refuge is no longer rapid or direct. The finding that vestibular lesions and hippocampal lesions disrupt 
dead reckoning suggests that the hippocampus may use vestibular information in dead reckoning. The vestibular system is important in maintaining the firing properties of place cells and head direction cells, and place cell firing can be modified by vestibular information (Sharp et al., 1995). Gothard et al. (2001) also demonstrated that place cell activity can be modified in the absence of visual information. This work adds to the growing literature that posits a role for the hippocampus in dead reckoning.

The use of vestibular information in dead reckoning may also contribute to the acquisition of piloting strategies (Dudchenko et al., 1997; Martin et al., 1997). A number of previous studies suggest that vestibular damage impairs learning of spatial problems (Potegal et al., 1977; Miller et al., 1983; Matthews et al., 1989; Semenov and Bures, 1989; Chapuis et al., 1992; Stackman and Herbert, 2002). The present study adds support to the suggestion that dead reckoning can contribute to the acquisition of accurate piloting. When the control and vestibular rats were tested from a new location, after receiving substantial experience from an old location, the control rats proceeded directly to the new location after finding that the home was no longer present at the old location (Whishaw and Tomie, 1997). This observation of "0-trial" learning suggests that the rats' accurate returns are mediated by dead reckoning. The vestibular rats required more trials to return to the new location than did the control rats, suggesting that they had to learn the new location using only piloting.

The loss of vestibular acuity resulting from disease or aging may contribute to spatial navigation impairments in humans. For example, patients with Meniere's disease who received unilateral vestibular neurotomy to eliminate vertigo are impaired in spatial inferences (short cutting home or reversing routes) immediately after the surgery (Peruch et al., 1999). In addition, Tetewsky and Duffy (1999) have observed that patients with Alzheimer's disease are impaired in radial patterns of optic flow perception. They found that this impairment was also associated with impaired ability to detect direction on a spatial navigation test.

An additional finding from the present experiments relates to the "emotionality" displayed by the rats after vestibular lesions. Although the rats were well trained on the foraging task before vestibular lesions, they displayed extreme reluctance to perform after the lesions and began to leave the refuge and search for food only after extensive additional training. This finding indicates that vestibular injury has quite a wide range of effects with respect to spatial behavior, even to the point of inducing a form of "agoraphobia." Indeed, there is a well documented connection between some forms of agoraphobia and vestibular dysfunction (Furman and Jacob, 2001; Jacob et al., 2001; Perna et al., 2001).

\section{REFERENCES}

Barlow JS (1964) Intertial navigation as a basis for animal navigation. J Theor Biol 6:76-117.

Barnes CA (1979) Memory deficits associated with senescence: a neurophysiological and behavioral study in the rat. J Comp Physiol Psychol 93:74-104.

Batschelet E (1981) Circular statistics in biology. London: Academic.

Chapuis N, Krimm M, De Waele C, Vibert N, Berthoz A (1992) Effect of post-training unilateral labyrinthectomy in spatial orientation task by guinea pigs. Behav Brain Res 51:115-126.

Chen YC, Pellis SM, Sirkin DW, Potegal M, Teitelbaum P (1986) Bandage backfall: labyrinthine and non-labyrinthine components. Physiol Behav 37:805-814.

Darwin C (1873) Origin of certain instincts. Nature 7:417-418.

Drai D, Benjamini Y, Golani I (2000) Statistical discrimination of natural modes of motion in rat exploratory behavior. J Neurosci Methods 96:119-131.

Dudchenko PA, Goodridge JP, Seiterle DA, Taube JS (1997) Effects of repeated disorientation on the acquisition of spatial tasks in rats: dissociation between the appetitive radial arm maze and aversive water maze. J Exp Psychol Anim Behav Process 23:194-210.

Etienne AS, Maurer R, Saucy F, Teroni E (1986) Short-distance homing in the golden hamster after a passive outward journey. Anim Behav 34:696-715.

Furman JM, Jacob RG (2001) A clinical taxonomy of dizziness and anxiety in the otoneurological setting. J Anxiety Disord 15:9-26.

Gallistel CR (1990) The organization of learning. Cambridge, MA: MIT.

Gothard KM, Hoffman KL, Battaglia FP, McNaughton BL (2001) Dentate gyrus and CA1 ensemble activity during spatial reference frame shifts in the presence and absence of visual input. J Neurosci 21:7284-7292.

Jacob RG, Whitney SL, Detweiler-Shostak G, Furman JM (2001) Vestibular rehabilitation for patients with agoraphobia and vestibular dysfunction: a pilot study. J Anxiety Disord 15:131-146.

Jarrard LE (1983) Selective hippocampal lesions and behavior: effects of kainic acid lesions on performance of place and cue tasks. Behav Neurosci 97:873-889.

Maaswinkel H, Jarrard LE, Whishaw IQ (1999) Hippocampectomized rats are impaired in homing by path integration. Hippocampus 9:553-561.

Martin GM, Harley CW, Smith AR, Hoyles ES, Hynes CA (1997) Spatial disorientation blocks reliable goal location on a plus maze but does not prevent goal location in the Morris maze. J Exp Psychol Anim Behav Process 23:183-193.

Matthews BL, Ryu JH, Bockaneck C (1989) Vestibular contribution to spatial orientation. Acta Otolaryngol 468:149-154.

Miller S, Potegal M, Abraham L (1983) Vestibular involvement in a passive transport and return task. Physiol Psychol 11:1-10.

Mittelstaedt ML, Mittelstaedt H (1980) Homing by path integration in a mammal. Naturwissenschaften 67:566-567.

Monnier M (1970) Function of the nervous system. New York: Elsevier.

Morris RG, Garrud P, Rawlins JN, O'Keefe J (1982) Place navigation impaired in rats with hippocampal lesions. Nature 297:681-683.

O’Keefe J, Nadel L (1978) The hippocampus as a cognitive map. Oxford: Clarendon.

Olton DS, Becker JT, Handelmann E (1979) Hippocampus, space, and memory. Behav Brain Sci 2:313-366.

Pellis SM, Pellis VC (1994) Development of righting when falling from a bipedal standing posture: evidence for the dissociation of dynamic and static righting reflexes in rats. Physiol Behav 56:659-663.

Pellis SM, Pellis VC, Teitelbaum P (1991) Labyrinthine and other supraspinal inhibitory controls over head-and-body ventroflexion. Behav Brain Res 46:99-102.

Pellis SM, Pellis VC, Nelson JE (1992) The development of righting reflexes in the pouch young of the marsupial Dasyurus hallucatus. Dev Psychobiol 25:105-125.

Perna G, Dario A, Caldirola D, Stefania B, Cesarani A, Bellodi L (2001) Panic disorder: the role of the balance system. J Psychiatr Res 35:279-286.

Peruch P, Borel L, Gaunet F, Thinus-Blanc G, Magnan J, Lacour M (1999) Spatial performance of unilateral vestibular defective patients in nonvisual versus visual navigation. J Vestib Res 9:37-47.

Potegal M (1982) Vestibular and neostriatal contributions to spatial orientation. In: Spatial abilities: development and physiological foundations (Potegal, M, ed), pp 361-387. New York: Academic.

Potegal M, Day MJ, Abraham L (1977) Maze orientation, visual and vestibular cues in two-maze spontaneous alternation of rats. Physiol Psychol 5:414-420.

Rawlins JN, Olton DS (1982) The septo-hippocampal system and cognitive mapping. Behav Brain Res 5:331-358.

Russell NA, Horii A, Liu P, Smith PF, Darlington CL, Bilkey DK (2000) Hippocampal place fields have decreased stability in rats with bilateral vestibular labyrinthectomies. Soc Neurosci Abstr 26:843.8.

Seguinot V, Maurer R, Etienne AS (1993) Dead reckoning in a small mammal: the evaluation of distance. J Comp Physiol [A] 173:103-113.

Semenov LV, Bures J (1989) Vestibular stimulation disrupts acquisition of place navigation in the Morris water tank task. Behav Neural Biol 51:346-363.

Sharp PE, Blair HT, Etkin D, Tzanetos DB (1995) Influences of vestibular and visual motion information on the spatial firing patterns of hippocampal place cells. J Neurosci 15:173-189.

Sirkin DW, Schallert T, Teitelbaum P (1980) Involvement of the pontine reticular formation in head movements and labyrinthine righting in the rat. Exp Neurol 69:435-457.

Stackman RW, Herbert AM (2002) Rats with lesions of the vestibular system require a visual landmark for spatial navigation. Behav Brain Res 128:27-40.

Stackman RW, Taube JS (1997) Firing properties of head direction cells in the rat anterior thalamic nucleus: dependence on vestibular input. J Neurosci 17:4349-4358.

Sutherland RJ, Whishaw IQ, Kolb B (1982) Spatial mapping: definitive 
disruption by hippocampal or medial frontal cortical damage in the rat. Neurosci Lett 31:271-276.

Taube JS (1998) Head direction cells and the neurophysiological basis for a sense of direction. Prog Neurobiol 55:225-256.

Techernichovski O, Benjamini Y, Golani I (1998) The dynamics of longterm exploration in the rat. Part I. A phase-plane analysis of the relationship between location and velocity. Biol Cybern 78:423-432.

Tetewsky SJ, Duffy CJ (1999) Visual loss and getting lost in Alzheimer's disease. Neurology 52:958-965.

Wallace DG, Hines DJ, Whishaw IQ (2002) Quantification of a single exploratory trip reveals hippocampal formation mediated dead reckoning. J Neurosci Methods 113:131-145.

Whishaw IQ, Gorny B (1999) Path integration absent in scent-tracking fimbria-fornix rats: evidence for hippocampal involvement in "sense of direction" and "sense of distance" using self-movement cues. J Neurosci 19:4662-4673
Whishaw IQ, Mittleman G (1986) Visits to starts, routes, and places by rats (Rattus norvegicus) in swimming pool navigation tasks. J Comp Psychol 100:422-431.

Whishaw IQ, Tomie J (1997) Piloting and dead reckoning dissociated by fimbria-fornix lesions in a rat food carrying task. Behav Brain Res 89:87-97.

Whishaw IQ, Coles BL, Bellerive CH (1995) Food carrying: a new method for naturalistic studies of spontaneous and forced alternation. J Neurosci Methods 61:139-143.

Whishaw IQ, Hines DJ, Wallace DG (2001) Dead reckoning (path integration) requires the hippocampal formation: evidence from spontaneous exploration and spatial learning tasks in light (allothetic) and dark (idiothetic) tests. Behav Brain Res 127:49-69.

Wiener S, Berthoz A (1993) Forebrain structures mediating the vestibular contribution during navigation. In: Multisensory control of movement (Berthoz A, ed), pp 427-456. New York: Oxford UP. 